\title{
Corrigendum to "Medicinal Properties and Active Constituents of Dracocephalum kotschyi and Its Significance in Iran: A Systematic Review"
}

\author{
Parisa Heydari, ${ }^{1,2}$ Maryam Yavari, ${ }^{1,2}$ Peyman Adibi $\left(\mathbb{D},{ }^{3}\right.$ Gholamreza Asghari, ${ }^{4}$ \\ Syed-Mustafa Ghanadian, ${ }^{5}$ Gabriel O. Dida, ${ }^{6,7}$ and Faham Khamesipour $\mathbb{D}^{8,9}$ \\ ${ }^{1}$ Department of Persian Medicine, Faculty of Medicine, Isfahan University of Medical Sciences, Isfahan, Iran \\ ${ }^{2}$ Department of Traditional Medicine, Isfahan University of Medical Sciences, Isfahan, Iran \\ ${ }^{3}$ Integrative Functional Gastroenterology Research Center, Isfahan University of Medical Sciences, Isfahan, Iran \\ ${ }^{4}$ Department of Pharmacognosy, School of Pharmacy and Pharmaceutical Sciences, Isfahan University of Medical Sciences, \\ Isfahan, Iran \\ ${ }^{5}$ Department of Pharmacognosy, Faculty of Pharmacy, Isfahan University of Medical Sciences, Isfahan, Iran \\ ${ }^{6}$ School of Public Health and Community Development, Maseno University, Kenya \\ ${ }^{7}$ Department of Community and Public Health, Technical University of Kenya, Nairobi, Kenya \\ ${ }^{8}$ Cellular and Molecular Research Center, Sabzevar University of Medical Sciences, Sabzevar, Iran \\ ${ }^{9}$ Shahid Beheshti University of Medical Sciences, Tehran, Iran
}

Correspondence should be addressed to Peyman Adibi; payman.adibi@gmail.com

and Faham Khamesipour; faham.khamesipour@yahoo.com

Received 10 July 2019; Accepted 10 July 2019; Published 21 July 2019

Copyright (C) 2019 Parisa Heydari et al. This is an open access article distributed under the Creative Commons Attribution License, which permits unrestricted use, distribution, and reproduction in any medium, provided the original work is properly cited.

In the article titled "Medicinal Properties and Active Constituents of Dracocephalum kotschyi and Its Significance in Iran: A Systematic Review" [1], Dr. Peyman Adibi should be listed as the second corresponding author.

\section{References}

[1] P. Heydari, M. Yavari, P. Adibi et al., "Medicinal properties and active constituents of dracocephalum kotschyi and its significance in Iran: A systematic review," Evidence-Based Complementary and Alternative Medicine, vol. 2019, Article ID 9465309, 14 pages, 2019. 


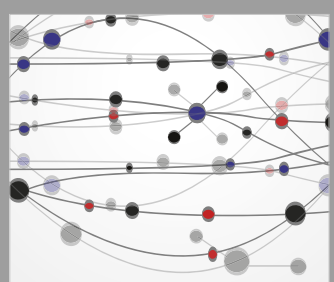

The Scientific World Journal
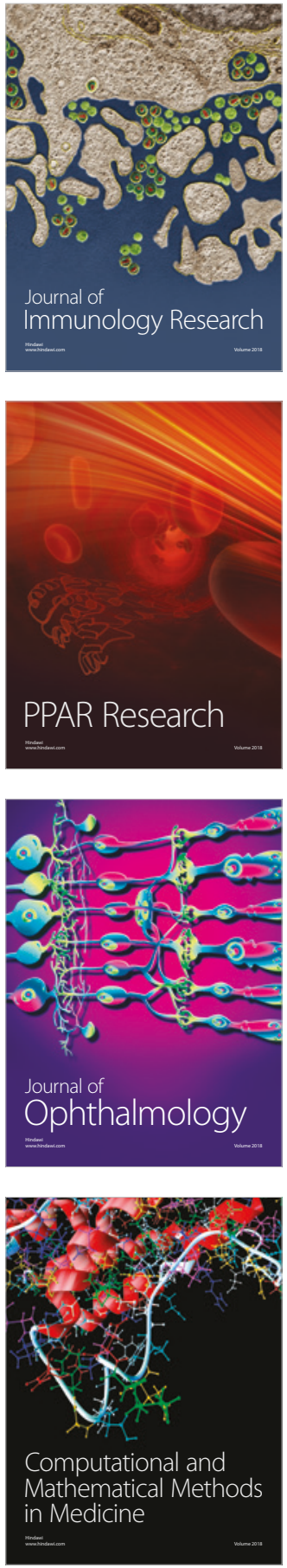

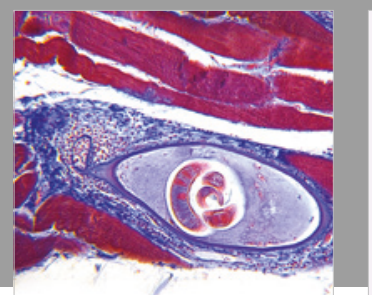

Gastroenterology Research and Practice

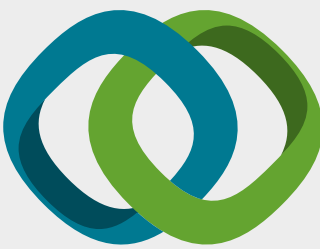

\section{Hindawi}

Submit your manuscripts at

www.hindawi.com
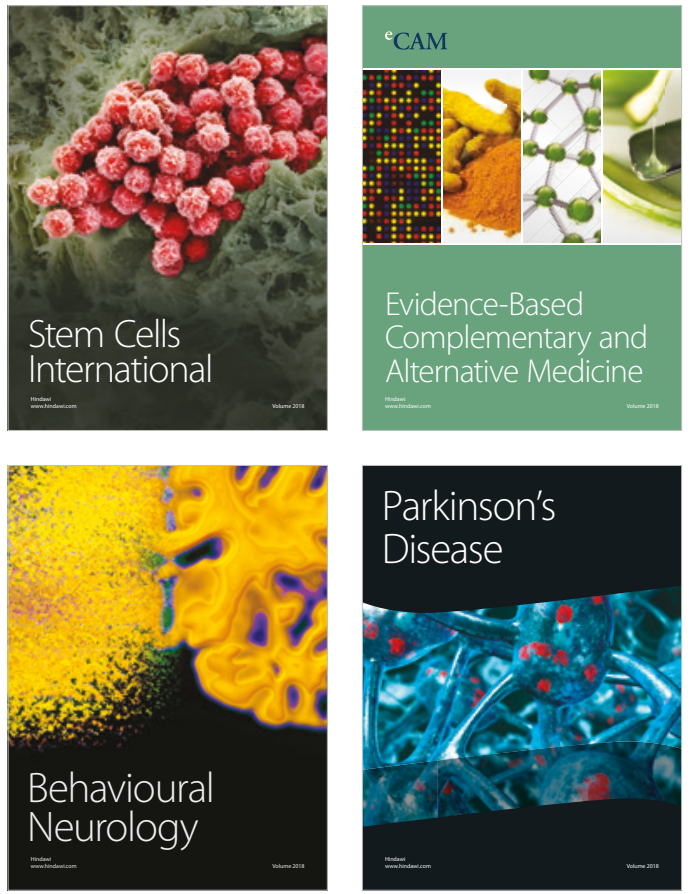

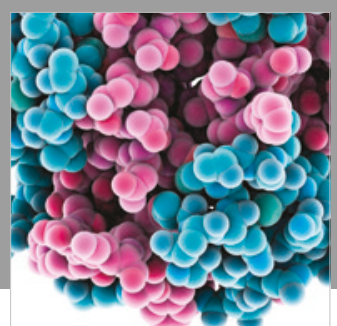

ournal of

Diabetes Research

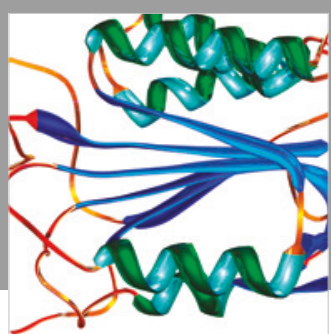

Disease Markers
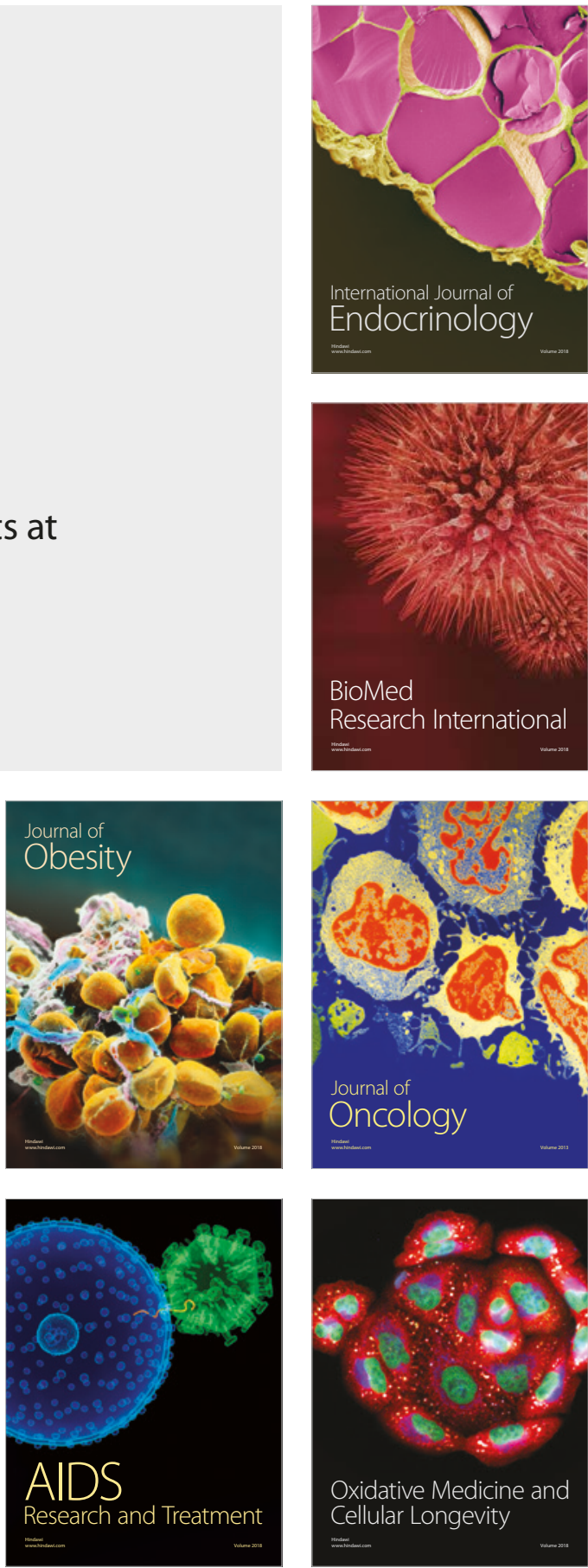